\title{
AVALIAÇÃO DA TOLERÂNCIA E NEFROTOXICIDADE DO ANTIMONIAL PENTAVALENTE ADMINISTRADO NA DOSE DE 40mg sbV/kg/DIA, DE 12/12h, POR 30 DIAS NA FORMA CUTÂNEO-MUCOSA DE LEISHMANIOSE
}

\author{
Raimunda N.R. Sampaio, Carmem Dea R. de Paula, João Herman D. \\ Sampaio, Rogério de S. Furtado, Pushkin P. Leal, Tânia T. Rosa, \\ Mario Ernesto Rodrigues e Joel Paulo R. Veiga
}

\begin{abstract}
Foi avaliada a função renal de 11 pacientes com leishmaniose cutâneo-mucosa tratados com antimonial pentavalente na dose de $40 \mathrm{mg} \mathrm{SbV} / \mathrm{kg} /$ dia aplicada de 12/12 horas, em esquema contínuo, durante trinta dias. No estudo, um paciente apresentou insuficiência renal reversível e dois desenvolveram alterações enzimáticas hepáticas e eletrocardiográficas sendo o esquema terapêutico interrompido. Nos demais pacientes observou-se efeitos nefrotóxicos tais como diminuição da taxa de filtração glomerular, diminuição da capacidade de concentração urinária, avaliada por um jejum hídrico de 16 horas e aumento na fração de excreção de sódio. No exame do sedimento urinário observou-se um aumento no número de leucócitos e cilindros. Os resultados encontrados neste estudo sugerem que o tratamento com antimonial pentavalente na dose de $40 \mathrm{mg} \mathrm{SbV/kg/dia} \mathrm{foi} \mathrm{menos} \mathrm{tolerado} \mathrm{em} \mathrm{virtude} \mathrm{de} \mathrm{seus} \mathrm{efeitos} \mathrm{tóxicos,} \mathrm{não}$ parecendo apresentar índice de cura superior ao esquema atualmente preconizado de $20 \mathrm{mg} \mathrm{SbV} / \mathrm{kg} / \mathrm{dia}$.
\end{abstract}

Palavras-chaves: Leishmaniose cutâneo-mucosa. Função renal. Antimonial pentavalente. Insuficiência renal.

A leishmaniose cutânea acomete cerca de 300.000 a 400.000 pessoas no mundo a cada ano 128 constituindo um problema na área de saúde pública em diversos países.

Os antimoniais pentavalentes permanecem como a base terapêutica e por causa da ocorrência de casos que não responderam aos esquemas descontínuos ou seriados de antimoniais buscou-se aplicá-los em esquemas inintemuptos7 9111518 27. Mesmo com a utilização de $20 \mathrm{mg}$ de $\mathrm{SbV} / \mathrm{kg} /$ dia de modo contínuo por 30 dias o percentual de cura clínica observado variou de 63 a $75 \%$ em doentes com forma mucosa acompanhados por um ano7 9 20. A existência de casos com resposta terapêutica insatisfatória aos antimoniais estimulou a busca de novas drogas ou mesmo a revisão das doses

Laboratório de Dermatomicologia e Laboratório de Nefrologia do Departamento de Clínica Médica da Faculdade de Ciências da Saúde e Hospital Universitário de Brasília. Brasília-DF. Apoio financeiro do CNPq (Projeto no 400345/90.0) e FAP-DF (Processo no 191000345/94).

Endereço para correspondência: Profa Raimunda Nonata Ribeiro Sampaio. Depto de Clínica Médica da Faculdade de Ciências da Saúde/UnB. Campus Universitário, Asa Norte, 70910-900 Brasília, DF. Fax: (061) 273-3907.

Recebido para publicação em 11/11/96. comumente prescritas dos mesmos13 16. A dose de 40mg de SbV/kg/dia já havia sido empregada em esquema seriado por Cucé e cols em 19826. Em 1987, Bryceson sugeriu esta dose como uma possibilidade terapêutica a ser empregada nos casos de leishmaniose mucosa3. Em doses padrões os antimoniais podem apresentar efeitos tóxicos manifestando-se com alteraç̃es eletrocardiográficas 4 , elevação de enzimas hepáticas18 20 e alterações da função renal24 2526.

Observamos, de modo a avaliar a eficácia e os efeitos colaterais com ênfase na nefrotoxicidade, 11 pacientes com forma cutâneo-mucosa, tratados com dose de 40mg de SbV/kg/dia (Glucantime ${ }^{\circledR}$ aplicada duas vêzes ao dia durante 30 dias.

\section{MATERIAL E MÉTODOS}

Foram incluídos no protocolo de investigação, pacientes de 18 a 60 anos de idade com diagnóstico confirmado de leishmaniose cutâneo-mucosa e que já haviam se submetido a tratamento com antimonial pentavalente na dose de $20 \mathrm{mg}$ de $\mathrm{SbV} / \mathrm{kg} /$ dia, durante 30 dias, sem êxito. Para a confirmação diagnóstica foram empregados dados da anamnese, epidemiologia 
Sampaio RNR, Paula CDR, Sampaio JHD, Furtado RS, Leal PP, Rosa TT, Rodrigues ME, Veiga JPR. Avaliação da tolerância e nefrotoxicidade do antimonial pentavalente administrado na dose de $40 \mathrm{mgSbv} / \mathrm{kg} / \mathrm{dia}$, de 12/12h, por 30 dias na forma cutâneo-mucosa de leishmaniose. Revista da Sociedade Brasileira de Medicina Tropical 30:457-463, nov-dez, 1997.

positiva e exames complementares, tais como intradermorreação de Montenegro, comprovação parasitológica, imunofluorescência indireta e histopatologia. Os critérios de exclusão foram gravidez e a existência de outras enfermidades hepáticas, renais ou cardíacas associadas.

Após a seleção os pacientes foram informados dos propósitos e do conteúdo do protocolo de pesquisa dando sua concordância por escrito em participar do estudo, segundo as normas que regulamentam as pesquisas em seres humanos12.

Os pacientes selecionados, em regime de internação hospitalar, foram submetidos por via EV a dose de 40mg de SbV/kg/dia, de 12/12 horas, não excedendo-se ao cálculo diário para um homem de $60 \mathrm{~kg}$. Foi realizado também, exame clínico completo, incluindo avaliação otorrinolaringológica.

Previamente, ao início da terapêutica e durante a mesma foi realizada a monitorização eletrocardiográfica duas vezes por semana e até diariamente conforme a necessidade. Avaliação laboratorial bioquímica com determinação da uréia, creatinina, enzimas hepáticas e exame sumário de unina foi realizada duas vezes por semana. Os critérios usados para a determinação da eficácia do esquema terapêutico foram a evolução clínica das lesões, complementada por critérios parasitológicos, sorológicos e histopatológicos. A função renal foi avaliada antes, aos 30 dias do uso do antimonial e uma semana após a sua suspensão. Estimou-se a filtração glomerular com a determinação da depuraçâo da creatinina endógena (DCE $\mathrm{ml} / \mathrm{min} / 1,73 \mathrm{~m}^{2}$ de SC). A função tubular distal foi avaliada através do teste de concentração urinária, com emprego de jejum hídrico de 16 horas, conforme descrito anteriormente 10.

Nas amostras de sangue e urina coletadas foram determinadas as concentrações de creatinina21, de sódio (fotometria de chama) e a osmolaridade (crioscopia, osmômetro Fiske). As depurações foram calculadas segundo fórmulas de uso corrente na literatura. $\mathrm{Na}$ análise estatística dos dados empregou-se o teste " $\mathrm{t}$ " de Student, comparando-se os valores obtidos com uma semana e aos trinta dias de uso da droga, com os valores basais observados antes do tratamento. Foi fixado o limite de significância de 5\%.

\section{RESULTADOS}

Todos os pacientes apresentaram intradermorreação de Montenegro positiva, 10 apresentaram comprovação parasitológica pela cultura, inoculação em hamster ou exame histopatológico; 9 pacientes tiveram imunofluorescência indireta positiva em títulos que variaram de 1:40 a 1:320 diluições. A cepa identificada por isoenzimas e anticorpos monoclonais em três destes pacientes, foi Leishmania Viannia braziliensis. Dos onze pacientes que iniciaram o esquema, em três 0 tratamento foi interrompido por aparecimento de efeitos colaterais e toxicidade. Em um paciente ocorreu hepatomegalia e elevação das enzimas hepáticas (TGO-190; TGP-150; fosfatase alcalina-17) e alargamento do intervalo Qtc até 0,51 segundos. No segundo paciente ocorreu insuficiência renal com uréia plasmática de $109 \mathrm{mg} \%$ e creatinina de $3,1 \mathrm{mg} \%$. No terceiro paciente ocorreu alargamento de espaço Q tc até 0,50 segundos. Após a recuperação destes doentes, os mesmos foram submetidos a outro esquema terapêutico com cura clínica de suas lesões. Nos oito pacientes que completaram os 30 dias de $40 \mathrm{mg}$ de $\mathrm{SbV} / \mathrm{Kg} /$ dia a função renal foi avaliada e os resultados estão expressos nas Tabelas 1,2 e 3 e Figuras 1 e 2.

Na Tabela 1, estão assinalados os valores da depuração de creatinina endógena, um teste que permite a estimativa aproximada da taxa de filtração glomerular. Verifica-se que houve uma queda significativa da depuração de creatinina aos trinta dias em relação ao período basal. Observa-se também que, após uma semana da suspensão do antimonial, a diminuição persiste de modo significativo, embora em termos médios os valores permaneceram na faixa normal (80$120 \mathrm{ml} / \mathrm{min} / 1,73 \mathrm{~m}^{2}$ de SC).

O estudo da capacidade de concentração urinária nos oito pacientes que completaram 0 tratamento está relatado nas Tabelas 2 a 3 e Figuras 1 e 2. Na Tabela 2 estão assinalados os valores do fluxo urinário (V), da osmolaridade urinária $\left(\mathrm{U}_{\mathrm{osm}}\right)$ e plasmática $\left(\mathrm{P}_{\mathrm{osm}}\right)$. Observase que aos trinta dias do uso do antimonial a $\mathrm{U}_{\mathrm{osm}}$ foi menor, de modo significativo, nos períodos 1 e 3 em relação aos valores basais correspondentes.

Na Tabela 3 podem ser observados os valores da depuração osmolar $\left(\mathrm{C}_{\mathrm{osm}}\right)$ e da depuração negativa de água livre $\left(\mathrm{TcH}_{2} \mathrm{O}\right)$. Observamos que os valores de $\mathrm{TcH}_{2} \mathrm{O}$ no período dois do teste realizado com trinta dias foi significativamente menor que o valor basal correspondente. 
Sampaio RNR, Paula CDR, Sampaio JHD, Furtado RS, Leal PP, Rosa TT, Rodrigues ME, Veiga JPR. Avaliação da tolerância e nefrotoxicidade do antimonial pentavalente administrado na dose de $40 \mathrm{mgSbv} / \mathrm{kg} / \mathrm{dia}$, de 12/12h, por 30 dias na forma cutâneo-mucosa de leishmaniose. Revista da Sociedade Brasileira de Medicina Tropical 30:457-463, nov-dez, 1997.

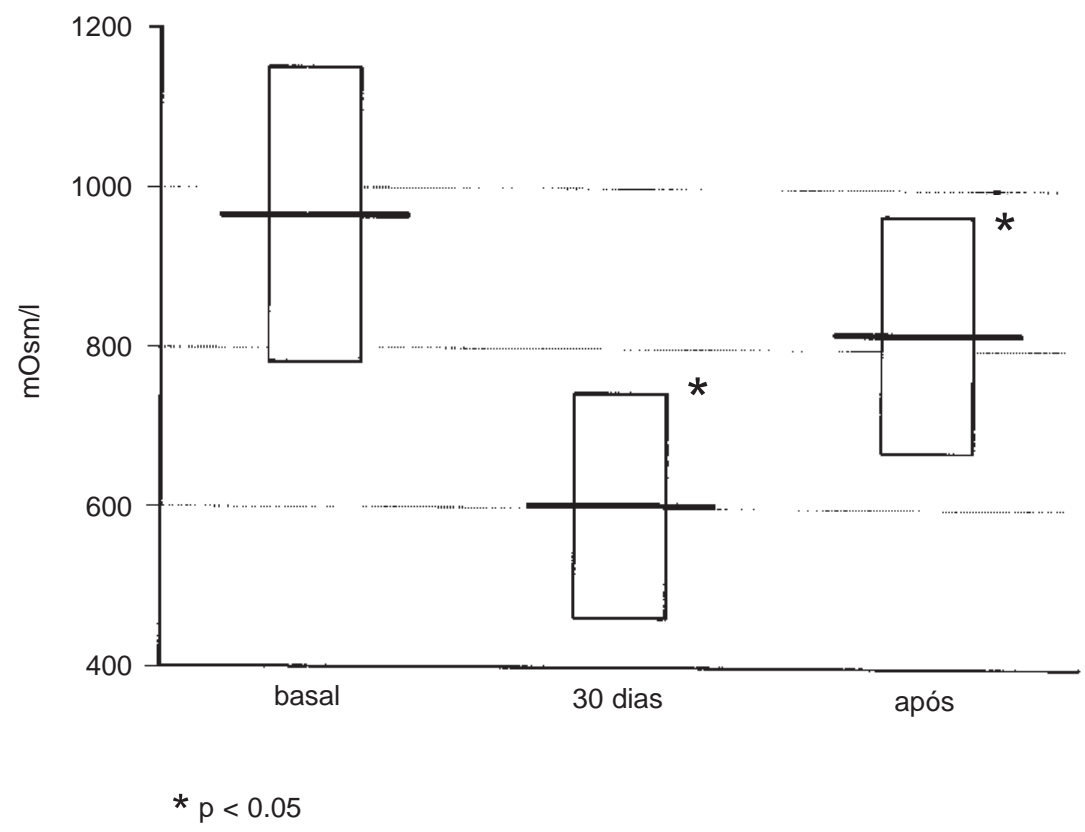

Figura 1 - Osmolaridade urinária máxima (mOsm/l). Valores expressos como média \pm desvio padrão.

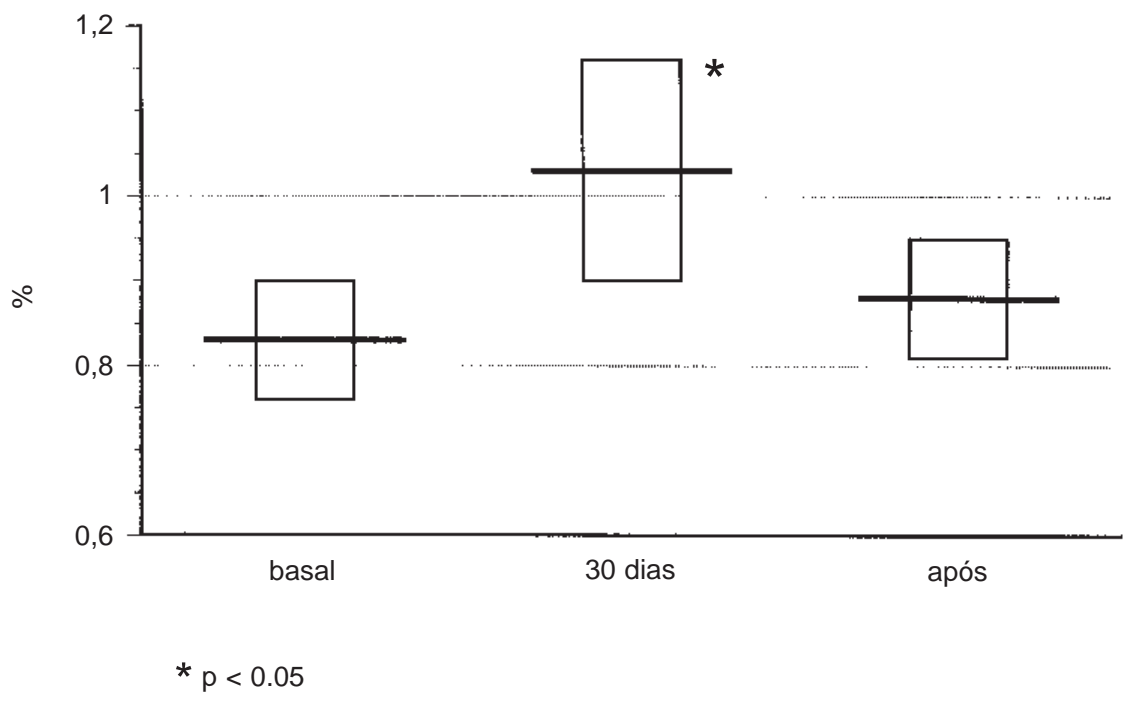

Figura 2 - Fração de excreção de sódio (\%). Valores expressos como média \pm desvio padrão.

\section{DISCUSSÃO}

Os percentuais de cura das formas visceral e cutâneo-mucosa da leishmaniose elevaramse com o uso dos antimoniais em esquemas terapêuticos ininterruptos 9 . Entretanto a falta de resposta terapêutica de algumas formas de leishmaniose, principalmente da forma mucosa 
Sampaio RNR, Paula CDR, Sampaio JHD, Furtado RS, Leal PP, Rosa TT, Rodrigues ME, Veiga JPR. Avaliação da tolerância e nefrotoxicidade do antimonial pentavalente administrado na dose de $40 \mathrm{mgSbv} / \mathrm{kg} / \mathrm{dia}$, de 12/12h, por 30 dias na forma cutâneo-mucosa de leishmaniose. Revista da Sociedade Brasileira de Medicina Tropical 30:457-463, nov-dez, 1997.

causada pela Leishmania Viannia braziliensis a esse novos esquemas, continua a constituir um problema sério no controle da doença3 91819 22, justificando a busca de novas estratégias terapêuticas com antimoniais e, ou 0 uso de novas drogas. Em virtude disto, Bryceson em 1987 propôs o uso do esquema de $40 \mathrm{mg} \mathrm{SbV} / \mathrm{kg} /$ dia usado em duas aplicações diárias, durante 30 dias, como possível solução terapêutica das formas mucosas de leishmaniose?

Os antimoniais pentavalentes, embora tenham rápida excreção renal14, quando utilizados em doses convencionais ou mais elevadas e por tempo mais prolongado, podem provocar alterações na função renal. Foram relatados o aparecimento de proteinúria17 e até casos de insuficiência renal aguda2 51719 .

Do ponto de vista funcional, Veiga e col. descreveram um distúrbio da função tubular renal em pacientes com leishmaniose cutâneo mucosa tratados com antimonial pentavalente, manifesto como diminuição da capacidade de concentração urinária, com preservação da função glomerular24 25. Estudo in vitro demonstrou um efeito do antimonial inibindo o fluxo de água osmoticamente induzido na preparação de bexiga de sapo, estrutura filogeneticamente semelhante ao túbulo coletor de mamífero. O pentacloreto de antimônio $\left(\mathrm{SbCl}_{5}\right)$ também exibiu este efeito e em doses altas foi tóxico para as células da bexiga, que não mais responderam à oxitocina 8 .

Estudo experimental no rato mostrou que 0 antimonial administrado na dose de $30 \mathrm{mg}$ de $\mathrm{SbV} / 100 \mathrm{~g}$ de peso/dia, por 30 dias provocou as alterações tubulares renais, sem afetar de modo significativo a função glomerular. Por outro lado, a dose de $200 \mathrm{mg} / 100 \mathrm{~g}$ de peso/dia por 30 dias alterou de modo significativo a taxa de filtração glomerular e aumentou a fração de excreção de sódio 23.

No presente estudo, dos onze pacientes com leishmaniose cutâneo-mucosa utilizando dose de $40 \mathrm{mg} \mathrm{SbV} / \mathrm{kg} /$ dia de antimonial pentavalente aplicada duas vezes ao dia, em esquema contínuo por 30 dias, observou-se que em três pacientes o esquema terapêutico foi suspenso por efeitos colaterais e toxicidade. Em oito pacientes que concluíram o tratamento, a resposta terapêutica mostrou que cinco pacientes obtiveram cura clínica das lesões, e em três houve falha terapêutica, resultado que parece não diferir do observado com o esquema na dose de $20 \mathrm{mg}$ de $\mathrm{SbV} / \mathrm{kg} / \mathrm{dia}$.
Os efeitos nefrotóxicos da dose de 40mg foram importantes, sendo que um paciente apresentou quadro de insuficiência renal aguda que reverteu com a suspensão do tratamento. Os valores estimados da taxa de filtração glomerular, através da depuração de creatinina endógena mostraram diminuição significativa aos 30 dias do uso do antimonial e com uma semana da suspensão da terapêutica, em relação ao período basal, embora com valores na faixa da normalidade e mostrando sinais de recuperação (Tabela 1). Também, nesta dose o antimonial provocou alterações funcionais tubulares importantes, expressas como uma diminuição significativa na capacidade de concentração urinária com tendência à normalização após a suspensão do tratamento (Tabelas 2 e 3 e Figura 1). No teste de jejum hídrico de 16 horas empregado, considera-se que a função de concentração urinária está preservada quando obtem-se pelo menos um valor de osmolaridade urinária de $800 \mathrm{mOsm} / 1$ em um dos três períodos do teste10. A fração de excreção de sódio, um índice de função tubular, elevou-se aos 30 dias de modo significativo, acima do valor normal que se situa em torno de 1\% (Figura 2). Este dado sugere que em doses elevadas o antimonial pentavalente pode determinar disfunção tubular renal. Somando-se aos achados funcionais, o exame do sedimento urinário mostrou uma elevação do número de leucócitos e aparecimento de cilindros, dados compatíveis com a nefrotoxicidade do medicamento.

Concluindo, os resultados do presente estudo sugerem que na dose de $40 \mathrm{mgSbV} / \mathrm{Kg} / \mathrm{dia}$, aplicada duas vezes ao dia, o antimonial pentavalente foi menos tolerado, do ponto de vista de seus efeitos nefrotóxicos, sem aparentemente apresentar índice de cura superior ao esquema atualmente preconizado de $20 \mathrm{mg} \mathrm{SbV/Kg/dia.}$

\section{SUMMARY}

The renal function of eleven patients with mucocutaneous leishmaniasis was analyzed in a prospective study realized at the School Hospital of University of Brasília. The patients were treated with doses of $40 \mathrm{mg} / \mathrm{kg} /$ day of pentavalent antimony $(\mathrm{SbV})$, in a continuous scheme during thirty days. In this study three patients were excluded, one patient with reversible renal failure and two patients with hepatic and cardiac malfunctions. In the other eight patients, severe nephrotoxics effects were observed, 
Sampaio RNR, Paula CDR, Sampaio JHD, Furtado RS, Leal PP, Rosa TT, Rodrigues ME, Veiga JPR. Avaliação da tolerância e nefrotoxicidade do antimonial pentavalente administrado na dose de $40 \mathrm{mgSbv} / \mathrm{kg} / \mathrm{dia}$, de 12/12h, por 30 dias na forma cutâneo-mucosa de leishmaniose. Revista da Sociedade Brasileira de Medicina Tropical 30:457-463, nov-dez, 1997.

like reduction of glomerular filtration rate, reduction of the urinary concentration capacity, evaluated by a sixteen hours hydric fasting and an increase of sodium fractional excretion. An increase in the number of leu cocytes and cylinders were observed at the urinary sediment exam. Finally, the results shows that the treatment with pentavalent antimony in doses of $40 \mathrm{mg} \mathrm{Sb} / \mathrm{kg} /$ day was less tolerated on account of its renal toxics effects. This scheme seems not be superior than the currently preconized scheme of $20 \mathrm{mg}$ of SbV/kg/day during 30 days.

Key-words: Mucocutaneous leishmaniasis. Renal function. Pentavalent antimony. Renal failure.

\section{AGRADECIMENTOS}

Ao técnico senhor Fernando V. de Pádua do Laboratório de Nefrologia da UnB, pela ajuda nas dosagens bioquímicas.

\section{REFERÊNCIAS BIBLIOGRÁFICAS}

1. Ashford RW, Desjeux P, De Raadt P. Estimation of population at risk of infection and number of cases of leishmaniasis. Parasitology Today 8:104105, 1992.

2. Balsan $M$, Fenech F. Acute renal failure in visceral leishmaniasis treated with sodium stibogluconate. Transactions of Royal Society of Tropical Medicine and Hygiene 86: 515-516, 1992.

3. Bryceson ADM. Therapy in man. In: Peters W, Killick-Kendrick $R$ (eds) The Leishmaniasis in biology and medicine, vol. 2, Academic Press, London, p. 847-907, 1987.

4. Chulay JD, Spencer HC, Mugambi M. Eletrocardiograph changes during treatment of leishmaniasis with pentavalent antimony (sodium stibogluconate). The American Journal of Tropical Medicine and Hygiene 34:702-709, 1985.

5. Cucé LC, Belda Júnior W, Dias MC. Alterações renais por hipersensibilidade ao uso de antimonial pentavalente (glucantime) na leishmaniose tegumentar americana. Relato de um caso. Revista do Instituto de Medicina Tropical São Paulo 32:249-251, 1990.

6. Cucé LC, Proença de FreitasTH, Prado de Oliveira ZN. Sampaio SAP. Tratamento da Leishmaniose tegumentar americana pelo antimonial pentavalente (Esquema rápido). Anais Brasileiros de Dermatologia 57:107-109, 1982.

7. Franke ED, Wignall FS, Cruz ME, Rosales E, Tovar AA, Lucas CM, Llanos-Cuentas A, Berman JD Efficacy and Toxicity of sodium stibogluconate for mucosal leishmaniasis. Annals of Internal Medicine 113:934-940, 1990.

8. Gagliardi ART, Veiga JPR, Rosa TT, Marsden PD. Pentavalent antimonial inhibition of the osmotic effect of oxytocin on the isolated toad bladder. Brazilian Journal of Medicine and Biological Research 18:567-571, 1985.

9. Herwaldt B, Berman JD. Recomendations for treating Leishmaniasis with sodium stibogluconate (Pentostan) and review of pertinent clinical studies. The American Journal of Tropical Medicine and Hygiene 46:296-306, 1992.

10. Kimachi T, Lomonaco AD, Gomes AU, Lima-Filho EC,Azevedo-Marques MM. Distúrbio do mecanismo de concentração urinária em pacientes com a forma crônica da moléstia de Chagas. Revista do Instituto de MedicinaTropical São Paulo 20:6-14, 1978.

11. Marsden PD, Sampaio RNR, Carvalho EM, Veiga JPR, Costa JLM, Llanos-Cuentas EA. High continuous antimony therapy in two pacients with unresponsive mucosal leishmaniasis. The American Journal of Tropical Medicine and Hygiene 34:710-713, 1985.

12. Ministério da Saúde - Declaração de Helsinki e Declaração da Assembléia Médica Mundial. In: Conselho de Organizações Internacionais de Ciências Médicas - OMS (ed) Diretrizes Internacionais propostas para Pesquisa Biomédica em seres humanos, Centro de Documentação do Ministério da Saúde, Brasília, DF, 1985.

13. Olliaro PL, Bryceson ADM. Practical Progress and new drugs for changing patterns of leishmaniasis. Parasitologic Today 9:323-328, 1993.

14. Rees PH, Kager PA, Keating MI, Hockmeyer WT. Renal clearance of pentavalent antimony (Sodium Stibogluconate). The Lancet 2:226-229, 1980.

15. Sampaio RN R. Tratamento Hospitalar da Leishmaniose Cutâneo-Mucosa.Tese de Mestrado, Universidade Federal de Minas Gerais, Belo Horinzonte, MG, 1984.

16. Sampaio RNR, Merchan EM, Vexenat A,Tristão RJ, Marsden PD. Combined antimonial and allopurinol therapy in mucosal leishmaniasis. Memórias do Instituto Oswaldo Cruz 85: 373-374, 1990.

17. Sampaio RNR, Rocha RAA, Marsden PD, Cuba CC, Barreto AC. Leishmaniose tegumentar americana, Casuística do Hospital Escola da UnB. Anais Brasileiros de Dermatologia 55:69-76, 1980.

18. Sampaio RNR, Sampaio JHD, Marsden PD. Pentavalent Antimonial treatment in mucosal leishmaniasis. The Lancet 1:1097, 1985. 
Sampaio RNR, Paula CDR, Sampaio JHD, Furtado RS, Leal PP, Rosa TT, Rodrigues ME, Veiga JPR. Avaliação da tolerância e nefrotoxicidade do antimonial pentavalente administrado na dose de $40 \mathrm{mgSbv} / \mathrm{kg} / \mathrm{dia}$, de 12/12h, por 30 dias na forma cutâneo-mucosa de leishmaniose. Revista da Sociedade Brasileira de Medicina Tropical 30:457-463, nov-dez, 1997.

19. Sampaio RNR, Veiga JPR, Limeira OM, Vexenat A, Marsden PD. Insuficiência renal aguda em leishmaniose tegumentar americana tratada com a associação de glucantime e alopurinol. Anais Brasileiros de Dermatologia 66:133-134, 1991.

20. Saenz RE, Rodrigues CG, Johnson CM, Berman JD. Efficacy and toxicity of Pentostan against Panamanian mucosal leishmaniasis. The American Journal of Tropical Medicine and Hygiene 44:394-398, 1991.

21. Slot C. Plasma creatinine determination. A new and specific Jaffe Reaction Method. Scandinavian Journal of Clinical Laboratory Investigation 17:381-387, 1965.

22. Thakur, CP. Harmful effect of high stibogluconate treatment of kala-azar in India.Transactions of the Royal Society of Tropical Medicine and Hygiene 80: 672-673,1986.

23. Veiga JPR, Khanan R, Rosa TT, Junqueira Jr LF, Brant PC, Raick AN, Friedman H, Marsden PD. Pentavalent antimonial nephrotoxicity in the rat. Revista do Instituto de Medicina Tropical São Paulo 32:304-309, 1990.
24. Veiga JPR, Rosa TT, Kimachi T, Wolff ER, Sampaio RNR, Gagliard ART, Junqueira Jr. LF, Costa JML, Marsden PD. Função renal em pacientes com leishmaniose muco-cutânea tratados com antimoniais pentavalentes. Revista do Instituto de Medicina Tropical São Paulo 27:298-302, 1985.

25. Veiga JPR, Wolff ER, Sampaio RNR, Marsden PD. Renal tubular dysfuction in patients with mucocutaneous leishmaniasis treated with pentavalent antimonials. The Lancet 3:359, 1983.

26. World Heath Organization. Report of the informal meeting on the chemotherapy of visceral leishmaniasis. Mimeographed document TDR/Chem-Leish/VL 82.3, 1982.

27. World Heath Organization. The Leishmaniases. Report of aWHO expert Comittee. Technical Report Series 701. Geneva, 1984.

28. World Heath Organization. Control of the Leishmaniasis. Reported of WHO expert Comittee. Technical Report Series 703. Geneva, 1990. 